\title{
Long noncoding RNA IncARSR confers resistance to Adriamycin and promotes osteosarcoma progression
}

\author{
Peng Shen ${ }^{1}$ and Yanfeng Cheng (1) ${ }^{1}$
}

\begin{abstract}
One of the significant challenges for chemotherapy is the appearance of resistance to compounds. Although several signaling pathways have been implicated in the development of Adriamycin (ADM) resistance, mechanisms involved in ADM-resistant osteosarcoma progression remain unknown. The present study attempted to illustrate the role of long noncoding RNA ARSR (IncARSR) in the development of adapted ADM resistance. We found IncARSR overexpressed in the Adriamycin-resistant cell lines U2OS/ADM and MG63/ADM, accompanied with acquired multidrug resistance against to paclitaxel and cisplatin. Overexpression of IncARSR triggered rhodamine 123 efflux and survival, as well as the migration of Adriamycin-resistant cells. Inversely, the depletion of IncARSR promoted rhodamine 123 retention and apoptosis, while reducing the motility of ADM-resistant cells. Further investigation revealed that the upregulation of IncARSR enhanced multidrug resistance-associated protein-1 (MRP1), apoptosis inhibitor Survivin, and matrix metalloproteinase-2 (MMP2) through activating AKT. The reduction of IncARSR overcame the resistance to ADM in U2OS/ADM mouse model. The current study gained novel evidence for understanding the mechanisms underlying adaptive ADM resistance and provided rationales to improve clinical outcomes of refractory osteosarcoma.
\end{abstract}

\section{Introduction}

Since the MAP (methotrexate, Adriamycin, and cisplatin) chemotherapy was introduced into the treatment for patients suffered from osteosarcoma, it is unchanged over the past decades ${ }^{1}$. Clinical outcomes, remaining $65-70 \%$, have similarly improved little over the last decades ${ }^{2}$. According to the development of immunotherapy and targeted therapy, patients received combination therapy, including surgical resection, chemotherapy, radiotherapy, and targeted therapy, are expected to prolong survival. Nevertheless, chemoresistance obstructs the response of relapse or metastasis of osteosarcoma to chemotherapy. There is an imperative need for verifying the details of chemoresistance.

Correspondence: Yanfeng Cheng (pumpkin0452@163.com)

'Department of Dermatology, Shengjing Hospital of China Medical University, 36 Sanhao Street, Heping District, Shenyang, Liaoning 110004, China

Edited by A. Stephanou
Long noncoding RNAs (lncRNAs) are RNA molecules with more than 200 nucleotides while lacking proteincoding capacity. Abnormal expression of lncRNAs is involved in dysregulation of different biological functions in cancer initiation, progression, and recurrence, such as proliferation, migration and invasion, stemness, radioresistance, and chemoresistance ${ }^{3}$. The mechanisms underlying different lncRNAs were discrepant. Previous studies have demonstrated that IncRNA FENDRR, LINC00161, and CTA enhanced sensitivity to active chemical agents ${ }^{4-6}$, whereas the others induced resistance via multiple signaling pathways.

LncRNAs play dual roles in regulating gene expression by various mechanisms. As oncogenes, the expression of H91antisense RNA $(91 \mathrm{H})$ elevated in osteosarcoma and correlated with osteosarcoma progression. Impairment of $91 \mathrm{H}$ reduced proliferation and promoted apoptosis ${ }^{7}$. MALAT-1, metastasis-associated lung adenocarcinoma transcript 1, enhanced PI3K/AKT activation and led to

\section{(c) The Author(s) 2020}

(c) (i) Open Access This article is licensed under a Creative Commons Attribution 4.0 International License, which permits use, sharing, adaptation, distribution and reproduction cc) in any medium or format, as long as you give appropriate credit to the original author(s) and the source, provide a link to the Creative Commons license, and indicate if changes were made. The images or other third party material in this article are included in the article's Creative Commons license, unless indicated otherwise in a credit line to the material. If material is not included in the article's Creative Commons license and your intended use is not permitted by statutory regulation or exceeds the permitted use, you will need to obtain permission directly from the copyright holder. To view a copy of this license, visit http://creativecommons.org/licenses/by/4.0/. 
osteosarcoma metastasis ${ }^{8}$. On the other hand, lncRNAs work as tumor suppressors. For instance, the negativefeedback loop of Loc285194 and miR-211 played an important role in osteosarcoma development ${ }^{9}$.

Recently, lncRNA Activated in RCC (renal cell carcinoma) with Sunitinib Resistance, lncARSR, was found to promote resistance against sunitinib in renal cancer ${ }^{10}$ and triggered tumor cell dissemination ${ }^{11}$. Furthermore, lncARSR enhanced hepatocellular carcinoma resistance to Adriamycin (ADM) and steatohepatitis via activation of $\mathrm{AKT}^{12,13}$. Besides, overexpression of lncARSR maintained cancer stem cells in liver cancer by activating STAT3mediated pathways ${ }^{14}$. Nevertheless, the knowledge of lncARSR in osteosarcoma is little. The present study found IncARSR expression raised in ADM-resistant osteosarcoma cells and aimed to illuminate the mechanisms underlying lncARSR increment and ADM resistance of osteosarcoma.

\section{Materials and methods Chemicals and reagents}

Adriamycin (Doxorubicin, ADM), paclitaxel (PTX), and cisplatin (DDP) were obtained from Selleck Chemicals (Houston, TX, USA). Lipofectamine RNAiMAX and dimethyl sulfoxide (DMSO) were purchased from Thermo Fisher Scientific (Waltham, MA, USA). siRNA negative control and siRNA targeting lncARSR were synthesized by OriGene. shRNA negative control and shRNA targeting lncARSR were inserted into pGFP-CshLenti vector and amplified according to the manufacturer's instructions (OriGene, Rockville, MD, USA).

\section{Cell culture}

Human osteosarcoma cell lines U2OS and MG63 were purchased from Cell Bank of the Chinese Academy of Sciences (Shanghai, China). Cells were cultured in RPMI1640 supplemented with 10\% fetal bovine serum (Thermo Fisher Scientific, Waltham, MA, USA) and were passaged upon reaching $70-80 \%$ confluency.

\section{Generation of Adriamycin-resistant cells}

U2OS and MG63 cells were exposed to intermittent and continue the stepwise increase of ADM concentrations from $0.2 \mathrm{mg} / \mathrm{l}$ ADM. Twenty-four hours later, ADM was removed, and cells were cultured with fresh medium until the proliferation rate returned to the identical rate of parental cells. At this point, the ADM concentrations increased and repeated the above process. During this 6-month-long period, the ADM-resistant subline of each cell line named $\mathrm{U} 2 \mathrm{OS} / \mathrm{ADM}$ and MG63/ADM were induced, respectively.

\section{LncRNA microarray analysis}

Total RNA from parental or resistant cells was used for RT2 LncRNA Array (QIAGEN N.V. Hilden, German).
LncRNA microarray analysis was performed according to the manufacturer's protocol. LncRNAs (fold change $>1.5$, and $P$-value $<0.05)$ were considered expressed distinctively between two groups. Three independent lncRNA arrays were performed. Data obtained from the lncRNA microarray was curated and approved by Gene Expression Omnibus (Accession no. GSE142230).

\section{Rhodamine 123 accumulation assay}

P-glycoprotein (P-gp) inhibitory potential was measured by the accumulation of rhodamine 123 in cells in the absence or presence of ADM. Details were described previously ${ }^{15}$. To measure the rhodamine 123 (Rh123) inhibitory potential of the ADM-resistant cells and their corresponding parental cells, $5 \times 10^{5}$ cells were seeded in 6-well plates. Twelve hours later, rhodamine 123 accumulation assay was performed following flow cytometry analysis. Data presented as a fold change of rhodamine 123-associated median fluorescence intensity (MFI).

\section{MTT assay}

Cell viability was determined by MTT assay, as previously mentioned ${ }^{16}$. Cells were plated with $0.1 \mathrm{ml}$ RPMI1640 supplemented $10 \%$ fetal bovine serum, together with DMSO or ADM. Ten microliters of MTT solution $(5 \mathrm{mg} /$ $\mathrm{ml}$ ) was complemented and followed by 4-h-long incubation before measurement. A Microplate Reader (Life Science, Hercules, CA, USA) was used to collect the optical densities at $490 \mathrm{~nm}$ (OD490nm). Data gained from three independent experiments. $\mathrm{IC}_{50}$ values were estimated as the drug concentration required for inhibiting $50 \%$ cell growth. Resistance index $(\mathrm{RI})=\mathrm{IC}_{50}$ of resistant cells $/ \mathrm{IC}_{50}$ of parental cells.

\section{JC-1 apoptosis analysis}

Cell apoptosis was analyzed by a JC- 1 assay kit (Beyotime, Shanghai, China) followed by flow cytometry (Beckham Coulter, Brea, CA, USA). Cells were plated in 6-well plates and allowed to attach overnight. JC-1 assay was conducted post-ARSR transfection $24 \mathrm{~h}$ according to the manufacturer's protocols. Results from three independent experiments were analyzed with Kaluza Analysis Software 2.1.1 (Beckham Coulter, Brea, CA, USA).

\section{Migration assay}

Transwell assay was performed using modified Boyden chambers without matrigel (Merck \& Co., Inc., Kenilworth, NJ, USA) to measure the cell migration. Cells in $0.2 \mathrm{ml}$ serum-free RPMI-1640 were seeded in the upper room of each chamber, while the lower room was filled with $0.6 \mathrm{ml}$ DMEM supplemented with 10\% FBS. After incubating for $24 \mathrm{~h}$ at $37^{\circ} \mathrm{C}$, cells on the upper compartments were removed, whereas the migrated cells in the lower parts were stained with $0.4 \%$ (w/v) Trypan blue 
solution, observed and counted under a $\times 100$ magnification microscope.

\section{Immunoblots}

Total proteins were extracted using $100 \mu \mathrm{l}$ lysis buffer form cells. After measurement of the quality and concentrations of the proteins, $25 \mu \mathrm{g}$ samples were loaded and separated in $10 \%$ sodium lauryl sulfatepolyacrylamide gel electrophoresis (SDS-PAGE). Proteins were transferred to nitrocellulose membranes through electroblotting. Then membranes were blocked with $5 \%$ skim milk for $1 \mathrm{~h}$ at room temperature, followed by incubation with P-gp (Cat. No. MA5-13854, Invitrogen, Carlsbad, CA, USA), MRP1 (SC-18835), Survivin (sc17779), MMP2 (sc-13594), pSer473-Akt (sc-293125), Akt1 (sc-5298), p-ERK (sc-7383), ERK (sc-514302), pSer33/37- $\beta$-catenin (sc-57535), $\beta$-catenin (sc-7963), p-NFKB (sc-271908), NFкB (sc-8414), p-mTOR (sc293089), mTOR (sc-517464), and GAPDH (SC-47724) antibodies (Santa Cruz Biotechnology, Inc., Dallas, TX, USA) overnight at $4{ }^{\circ} \mathrm{C}$ in a dilution of $1: 1000$. Membranes were washed three times with phosphate-buffered saline Tween-20 and incubated with HRP-conjugated secondary antibody (Merck \& Co., Inc., Kenilworth, NJ, USA) for another $1 \mathrm{~h}$ in a dilution of 1:2500. Immunoreactivity was detected using the Western Lighting Ultra (Thermo Fisher Scientific, Waltham, MA, USA).

Quantitative real-time polymerase chain reaction (qRT-PCR)

Total RNA was isolated with an RNA Isolation Kit (QIAGEN N.V. Hilden, German) according to the manufacturer's protocol. One milligram RNA was reverse transcribed to cDNA by QuantiTect Reverse Transcription Kit (QIAGEN N.V. Hilden, German). Real-time PCR was carried out with the Mx3000P real-time PCR system (Thermo Fisher Scientific, Waltham, MA, USA). The protocols were as follows: 40 cycles of $94{ }^{\circ} \mathrm{C}$ for $15 \mathrm{~s}, 60^{\circ} \mathrm{C}$ for $10 \mathrm{~s}$, and $72{ }^{\circ} \mathrm{C}$ for $20 \mathrm{~s}$. All procedures repeated thrice. Gene expression was normalized to the GAPDH to calculate relative expression using the $2^{-\Delta \Delta C \mathrm{q}}$ method $^{17}$. Primers for detecting lncARSR, MRP1, SURVIVIN, MMP2 $A K T$, and $m T O R$, listed below:

LncARSR, forward: 5'-TTTGAAATGCTCTTTGAGG GAT-3'; reverse: 5'-TGCAGGTTGTCTGAAGTTGGA3'. MRP1, forward: 5'-ACCCTAATCCCTGCCCAGAG3', reverse: 5'-CGCATTCCTTCTTCCAGTTC-3'. SURVIVIN, forward: 5'-AAGAACTGGCCCTTCTTGGA-3', reverse: 5'-CAACCGGACGAATGCTTTT. $M M P 2$, forward: 5'-AGCGAGTGGATGCCGCCTTTAA-3', reverse: 5'-CATTCCAGGCATCTGCGATGAG-3'. AKT, forward: 5'-TGGACTACCTGCACTCGGAGAA-3', reverse: 5'-GT GCCGCAAAAGGTCTTCATGG-3'. MTOR, forward: 5'-A GCATCGGATGCTTAGGAGTGG-3', reverse: 5'-CAG CCAGTCATCTTTGGAGACC-3'. GAPDH, forward:
5'-GTCTCCTCTGACTTCAACAGCG-3', reverse: 5'-AC CACCCTGTTGCTGTAGCCAA-3'.

\section{Generation of U2OS/ADM human osteosarcoma mouse models}

All studies were approved by the medical ethical committee of Shengjing Hospital of China Medical University and conducted according to the guidelines of the Centre of Experimental animal of Shengjing Hospital of China Medical University. Four-week-old female BALB/c nude mice were purchased from Nanjing Biomedical Research Institute of Nanjing University. $3 \times 10^{6} \mathrm{U} 2 \mathrm{OS} / \mathrm{ADM}$ cells expressing shRNA negative control or shRNA targeting lncARSR in $200 \mu \mathrm{l}$ saline were injected into nude mice subcutaneously. Seven days later, 24 mice with $\sim 100-\mathrm{mm}^{3}$ tumors were grouped randomly (six per group) and received $6 \mathrm{mg} / \mathrm{kg} \mathrm{ADM}$ or saline by intraperitoneal injection once weekly. The body weights of the mice, as well as the tumor volumes, were measured every 4 days. The tumor volume was estimated using the formula $V=$ $1 / 2$ (width ${ }^{2} \times$ length). The in vivo experiments terminated at day 28 post-treatment with ADM and mice were euthanasia. Tumors were resected and weighed. Immunohistochemical staining was performed followed the standard procedure ${ }^{18}$.

\section{Statistical analysis}

The data from all experiments were shown as means plus standard deviation. The differences were evaluated by one-way analysis of variance (ANOVA) with LSD test, and $P<0.05$ was statistically significant. Statistical analysis was conducted using GraphPad version 7.0 (San Diego, CA, USA).

\section{Results}

Establishment of Adriamycin-resistant osteosarcoma cells

To evaluate the resistance to ADM, we calculated the resistance index (RI) by estimating the ratio of the halfmaximal inhibitory concentration $\left(\mathrm{IC}_{50}\right)$ of ADMresistant cells over parental cells. Table 1 exhibited the

Table $1 I_{50}$ and $R I$ values of the indicated cell lines against Adriamycin.

\begin{tabular}{lrl}
\hline Cell line & \multicolumn{1}{c}{ IC $_{\mathbf{5 0}}$} & RI \\
\hline U2OS & $0.14 \pm 0.03$ & 1 \\
U2OS/ADM & $13.38 \pm 0.54$ & 95.57 \\
MG63 & $0.18 \pm 0.02$ & 1 \\
MG63/ADM & $13.12 \pm 1.11$ & 72.89 \\
\hline
\end{tabular}

Unit: $\mu \mathrm{mol} / \mathrm{l}$

$\mathcal{I}_{50}$ the half-effective inhibition concentrations, $R I$ resistance index. 

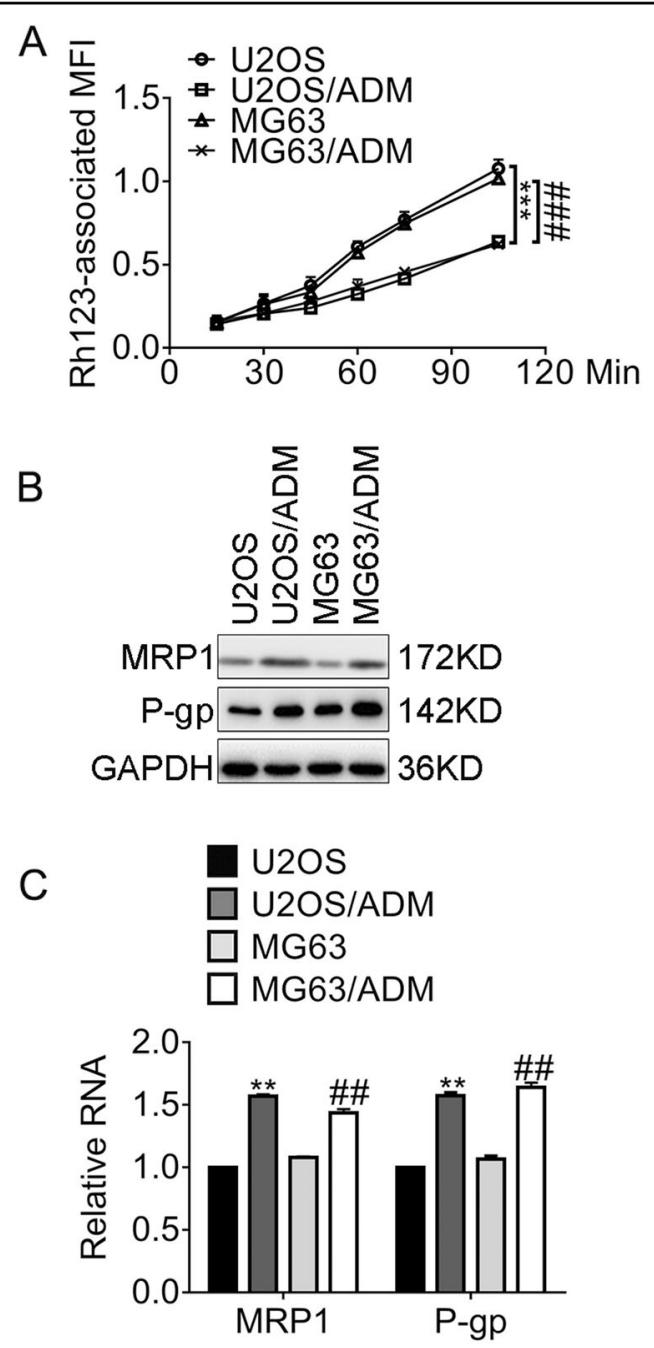

Fig. 1 Resistance profiles of Adriamycin-resistant cells. a The rhodamine 123-associated median fluorescence intensity (MFI) of the indicated cells. ${ }^{* *} P<0.001$, vs. U2OS; ${ }^{\# \#} P<0.001$, vs. MG63. b The expression of the indicated proteins was accessed by immunoblots. c The expression of the indicated genes was accessed by qRT-PCR analysis. ${ }^{*} P<0.01$, vs. U2OS; ${ }^{\# \#} P<0.01$, vs. MG63. Data obtained from at least three independent experiments and presented as means plus standard deviation.
$\mathrm{IC}_{50}$ and the corresponding RI values of each cell line. The RI values of U2OS/ADM and MG63/ADM increased by 95- and 72-fold, respectively. Because of the frequent cross-resistance to different agents of chemo-resistant osteosarcoma cells, we further conducted MTT assay with ADM-resistant cells exposed to paclitaxel (PTX) and cisplatin (DDP). Table 2 showed the $\mathrm{IC}_{50}$ and the corresponding RI values of each cell line against PTX and DDP. The RI values of U2OS/ADM against PTX and DDP were 3.01- and 3.06-fold stronger than the parental cell line at the $\mathrm{ID}_{50}$ level, separately. Similarly, the RI values of MG63/ADM against PTX and DDP were 2.95 and 3.03, separately. The above results revealed that the ADMresistant cells gained multidrug resistance (MDR). To determine the P-glycoprotein (P-gp) inhibitory potential, we selected the parental osteosarcoma cells and the ADM-resistant cells to perform the rhodamine 123 accumulation assay. Figure 1a exhibited the rhodamine 123-associated median fluorescence intensity (MFI) of U2OS/ADM cells decreased significantly compared to U2OS cells $(0.64$ vs. $1.08, P<0.001)$. Likewise, the Rh123associated MFI of MG63/ADM reduced markedly in comparison with MG63 cells $(0.62$ vs. $1.62, P<0.001)$. According to the identical efflux curves, the activity of Pgp increased in ADM-resistant cells compared with that in the parental cells. Therefore, we explored the multidrug resistance-associated protein-1 (MRP1) and the apoptosis inhibitor Survivin in the parental- and ADM-resistant cells. Figure 1b and c showed that MRP1 and P-gp was upregulated in U2OS/ADM at protein level and mRNA level, separately. Similar results were observed in MG63/ ADM cells compared to MG63 cells. In brief, the results displayed the resistance profiles of U2OS/ADM and MG63/ADM cells.

\section{LncRNA ARSR increases in ADM-resistant osteosarcoma cells and enhances cell survival exposed to ADM}

In the light of the analysis of lncRNA microarray data, we found several distinctly expressed lncRNA in U2OS/ ADM cells (Fig. 2a), including lncARSR. Consistent with the results from microarray, the expression of lncARSR

Table $2 \quad I_{50}$ and RI values of the indicated cell lines against PTX or DDP.

\begin{tabular}{llllllll}
\hline & \multicolumn{1}{l}{ IC50 } & & & & RI & \\
\cline { 2 - 6 } & U2OS & U2OS/ADM & MG63 & MG63/ADM & & U2OS/ADM & MG63/ADM \\
\hline PTX & $3.48 \pm 0.12$ & $10.46 \pm 0.04$ & $4.11 \pm 0.24$ & $12.14 \pm 0.15$ & 3.01 & 2.95 \\
DDP & $4.07 \pm 0.81$ & $12.47 \pm 0.72$ & $5.07 \pm 0.32$ & $15.37 \pm 0.42$ & 3.06 & 3.03 \\
\hline
\end{tabular}

Unit: $\mu \mathrm{mol} / \mathrm{l}$.

$I_{50}$ the half-effective inhibition concentrations, $R /$ resistance index. Unit, $\mu \mathrm{mol} / \mathrm{l}$.

PTX paclitaxel, DDP cisplatin. 

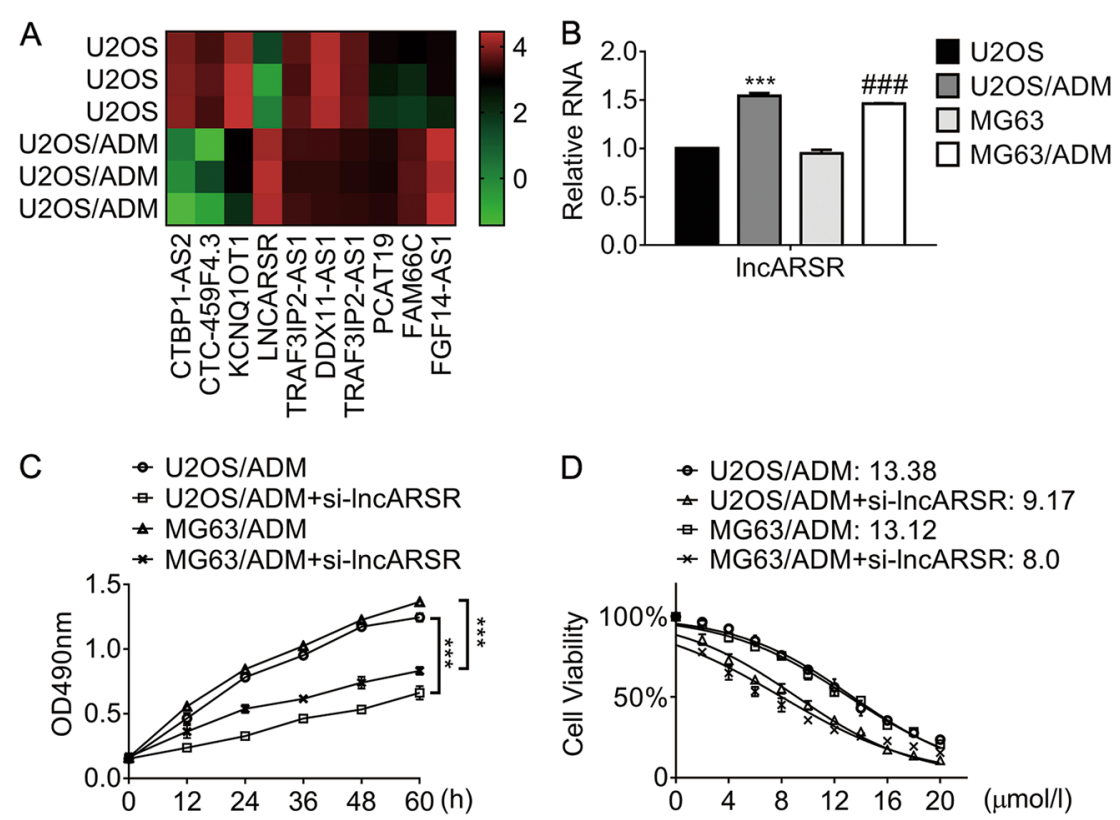

Fig. 2 LncARSR increases in Adriamycin-resistant cells and relates to resistance. a The heatmap showed the distinct expression of IncRNA in U2OS and U2OS/ADM cells. $\mathbf{b}$ The expression of IncARSR in ADM-resistant cells and parental cells was examined by qRT-PCR. ${ }^{* * *} P<0.001$, vs. U2OS;

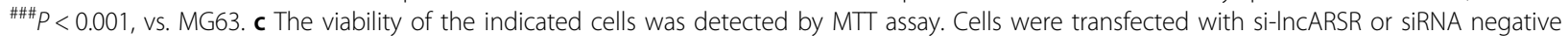
control, and MTT assay was conducted at different time-points. ${ }^{* *} P<0.001$, vs. U2OS/ADM or MG63/ADM cells, respectively. $\mathbf{d}$ The viability of the indicated cells post-transfection of si-IncARSR or siRNA negative control was accessed by MTT assay. The half-effective inhibition concentrations (IC $\left.C_{50}\right)$ were calculated according to the data obtained from three independent MTT assays. Data obtained from at least three independent experiments and presented as means plus standard deviation.

raised apparently in ADM-resistant cells compared with the corresponding parental cells (Fig. 2b). Moreover, knockdown of lncARSR in U2OS/ADM cells suppressed cell growth evidently compared to that expressing scrambled siRNA (Fig. 2c) in a time-dependent manner. In addition, the downregulation of IncARSR in the resistant cells reduced the $\mathrm{IC}_{50}$ values against $\mathrm{ADM}$. As the results shown in Fig. 2 d, the $\mathrm{IC}_{50}$ values of U2OS/ADM expressing si-lncARSR decreased by $30 \%$ approximately, and the $\mathrm{IC}_{50}$ values of MG63/ADM expressing silncARSR dropped by $40 \%$ compared with their parental cells. The results in Fig. 2 indicated that lncARSR was essential for the resistance to ADM.

\section{Overexpression of IncARSR promotes the viability and migration while antagonizes apoptosis of osteosarcoma cells}

To investigate the role of lncARSR in resistance against ADM, we introduced lncARSR into U2OS and MG63 cells. The viability of cells expressing lncARSR was accessed by MTT assay afterward. Figure 3a showed that overexpression of IncARSR promoted viability of U2OS and MG63 cells compared to control. Furthermore, the percentage of apoptotic U2OS cells decreased from 20.01 to $2.05 \%$ in the presence of ADM (Fig. 3b). Consistently, the results in Fig. 3c showed the apoptotic MG63 cells expressing lncARSR fell significantly post-exposure to ADM (control, $17.08 \%$ vs. IncARSR, 1.93\%). In addition, Fig. 3d demonstrated that the median counts of migrant U2OS cells expressing lncARSR increased apparently (lncARSR, 66 vs. control, 44). Similarly, Fig. 3e exhibited the number of migrated MG63 cells raised from 26 (control) to 51 (lncARSR). To explore the mechanisms underlying the alterations, we determined the expression of MRP1, Survivin and matrix metalloproteinase-2 (MMP2) by immunoblots and qRT-PCR, separately. The results in Figs. $3 \mathrm{f}$ and g showed the ectopic expression of lncARSR triggered expression of MRP1, Survivin, and MMP2 both at protein- and mRNA levels.

\section{Downregulation of IncARSR hampers the viability and motility while inducing rhodamine 123 accumulation and apoptosis of ADM-resistant cells}

Apart from gain-of-function experiments, we carried out loss-of-function experiments to evaluate the effects of lncARSR on ADM resistance. The viability of resistant cells lacking lncARSR was determined by MTT assay. As shown in Fig. 4a, the optical density at $490 \mathrm{~nm}$ fell apparently in cells expressing siRNA targeting lncARSR cells while that expressing siRNA negative control remained stable in an ADM doses-dependent manner. Similar results were gained in MG63/ADM cells. 

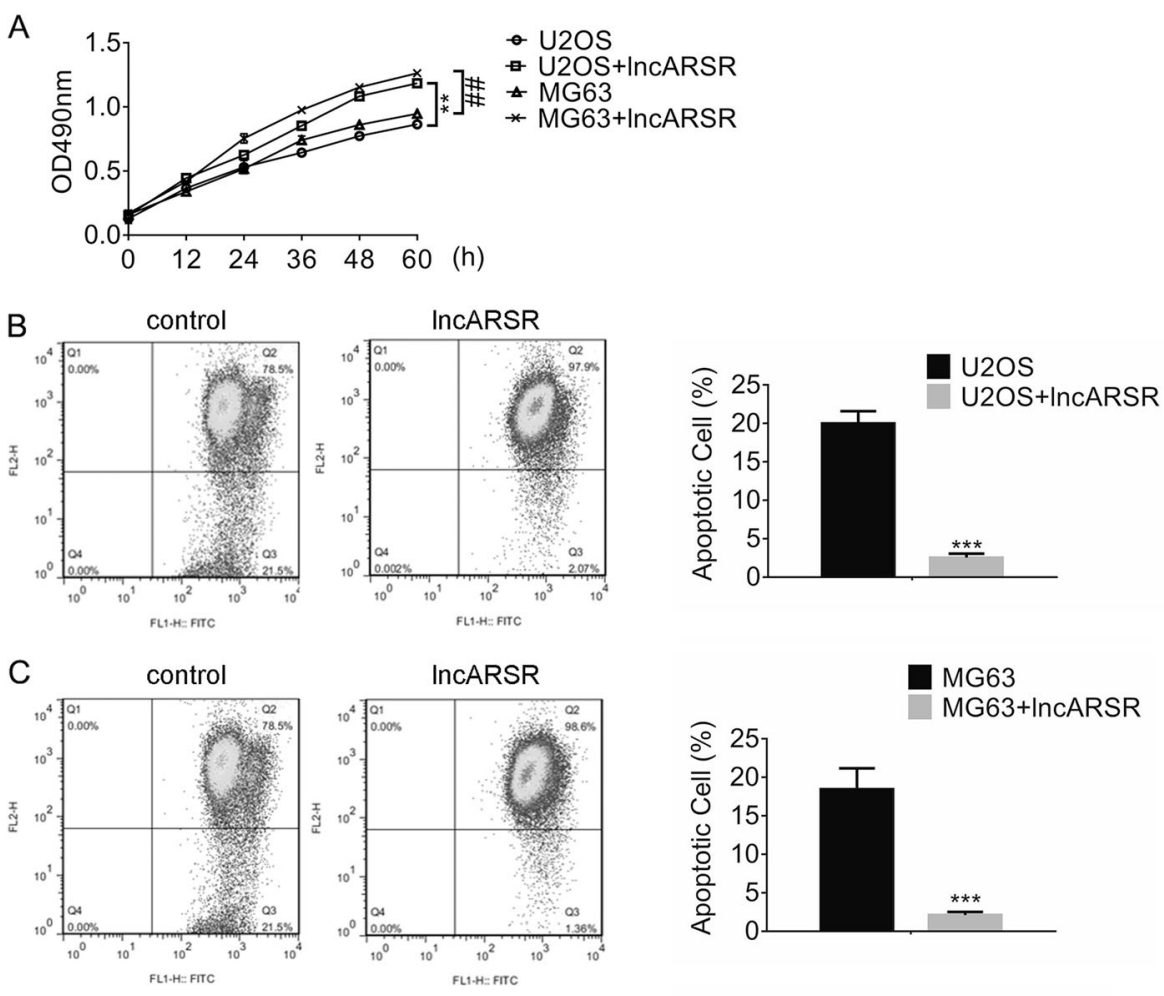

D
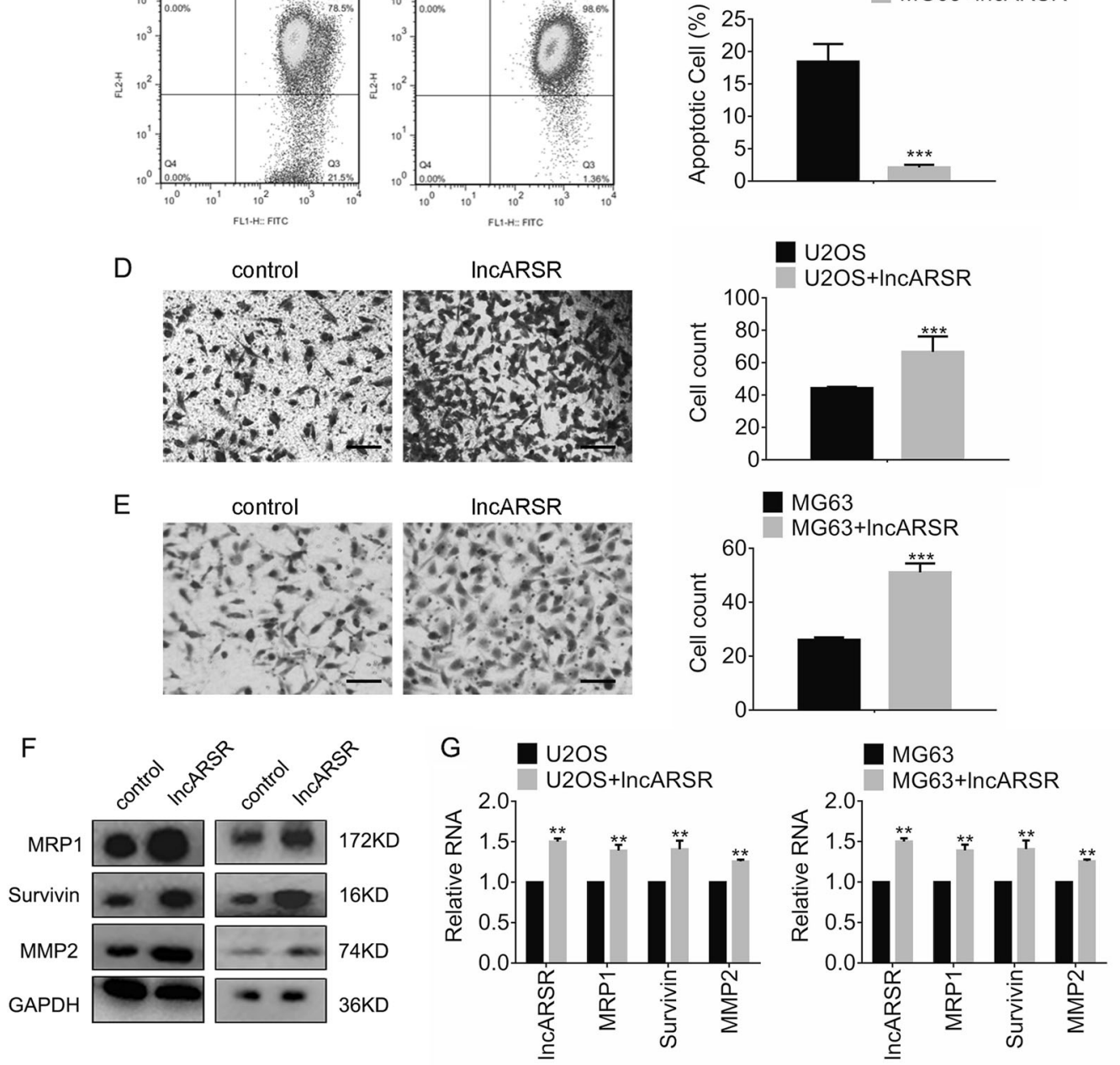

Fig. 3 Overexpression of IncARSR promotes the viability and migration of the parental osteosarcoma cells, while antagonizes apoptosis. a The viability of cells expressing control or LncRNA ARSR was determined by MTT assay. ${ }^{* *} P<0.01$, vs. U2OS plus control; ${ }^{\#} P<0.01$, vs. MG63 plus control. Apoptosis of (b) U2OS and (c) MG63 cells expressing control or LncARSR was accessed by JC-1 assay following by flow cytometry analysis. ${ }^{* * *} P<0.001$, vs. control. Migration of (d) U2OS and (e) MG63 cells were detected by transwell assay. ${ }^{* *} P<0.001$, vs. control. Scale bar, $200 \mu \mathrm{m}$. Magnification, $\times 100$. $\mathbf{f}$ The indicated protein expression in U2OS and MG63 cells were analyzed by immunoblots. $\mathbf{g}$ The expression of the indicated IncRNA and mRNA in U2OS and MG63 cells were analyzed by qRT-PCR. ${ }^{* *} P<0.01$, vs. control. Data obtained from at least three independent experiments and presented as means plus standard deviation. 

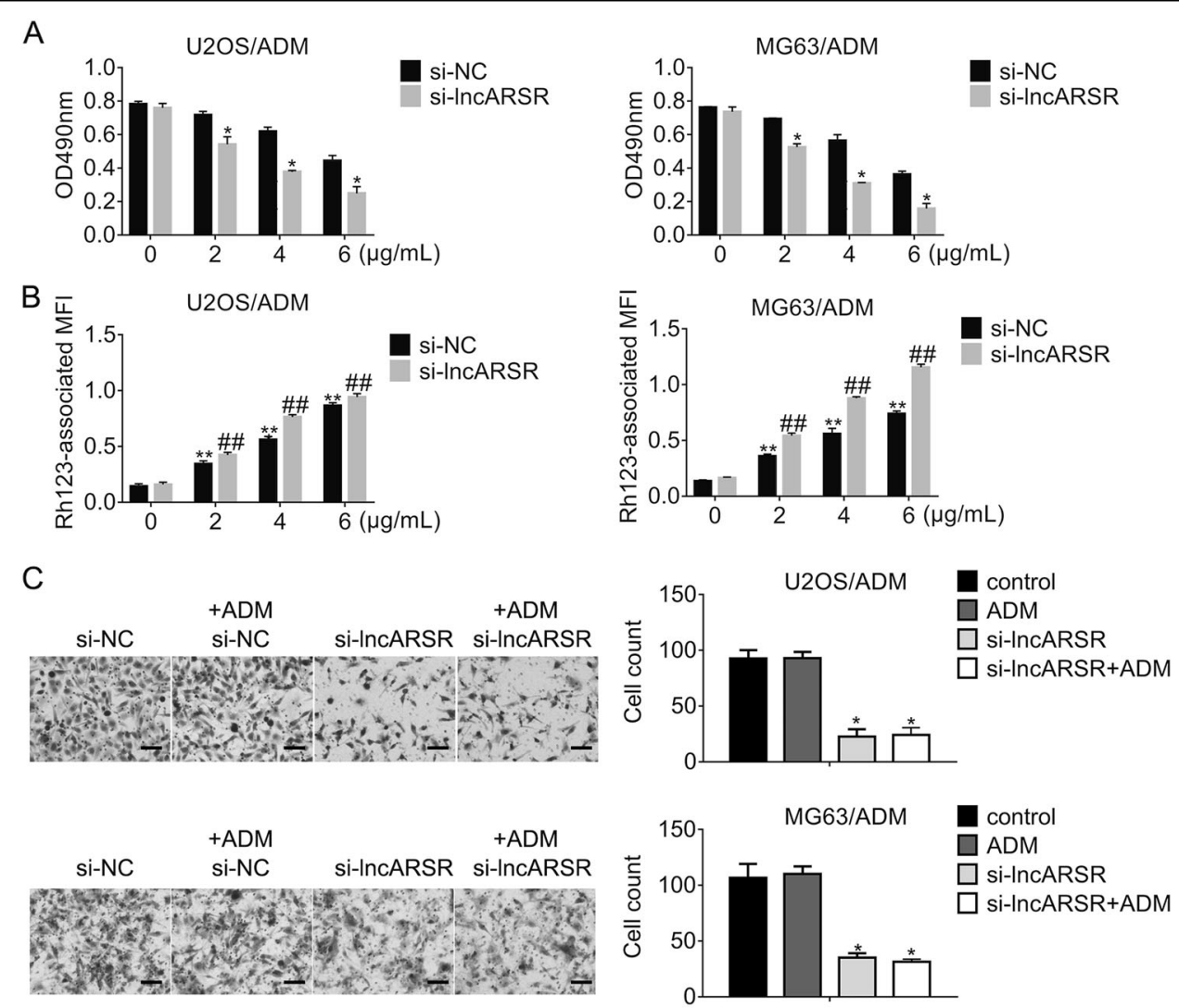

$\mathrm{D}$

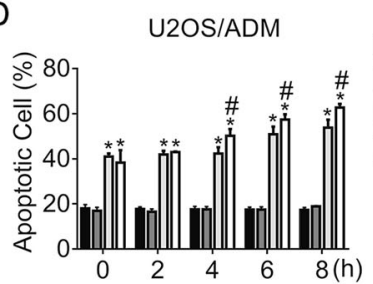

si-NC

$\square$ si-NC+ADM $\square$ si-InCARSR

$\square$ si-IncARSR+ADM

E

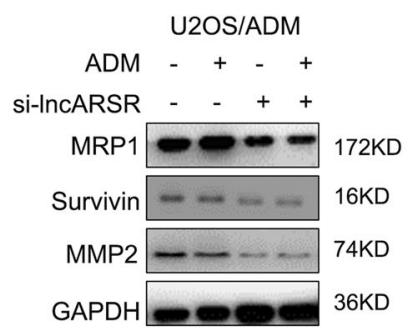

F

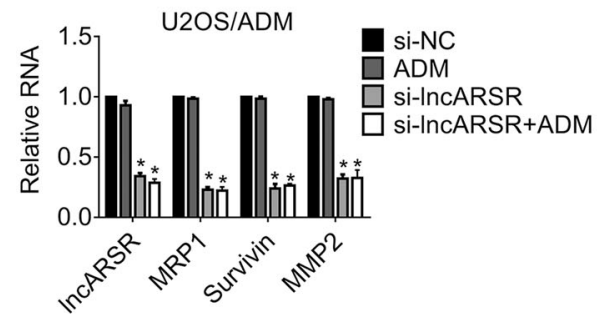

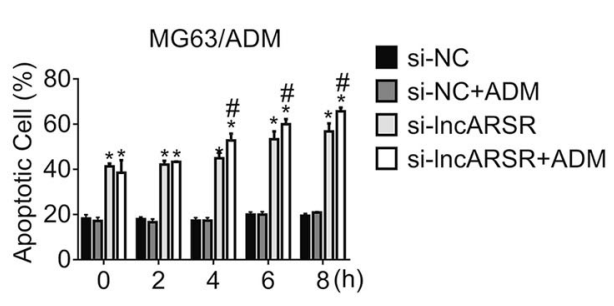

MG63/ADM
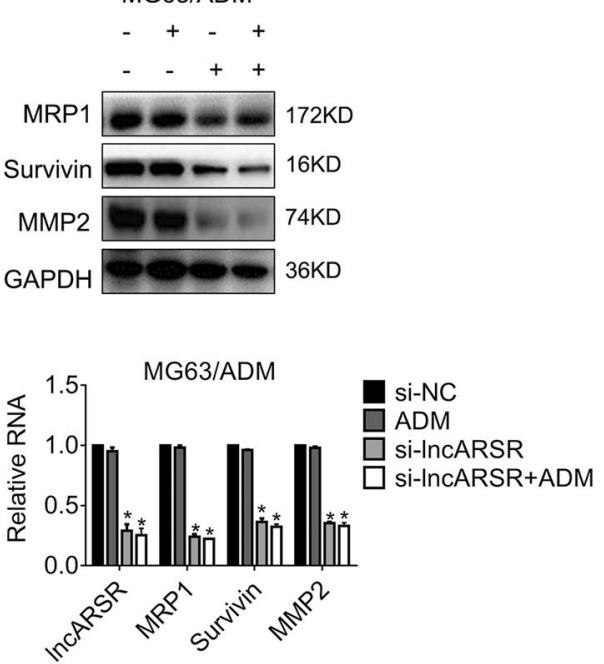

Fig. 4 (See legend on next page.) 
(see figure on previous page)

Fig. 4 Reduction of IncARSR impairs the growth, rhodamine 123 efflux, and migration of the Adriamycin-resistant cells while enhancing apoptosis. a Cell viability of U2OS/ADM or MG63/ADM cells expressing scramble siRNA or si-IncARSR was detected by MTT assay. ${ }^{*} P<0.05$, vs. si-NC. b Rh123 retention of U2OS/ADM and MG63/ADM cells expressing si-NC or si-IncARSR was accessed by rhodamine 123 assay and flow cytometry analysis. ${ }^{* *} P<0.01$, vs. si-NC without ADM. ${ }^{\# \#} P<0.01$, vs. si-NC plus ADM. c The migration of U2OS/ADM cells were detected by transwell assay, respectively. ${ }^{*} P<0.05$, vs. Si-NC. Scale bar, $200 \mu$ m. Magnification, $\times 100$. $\mathbf{d}$ The apoptosis of U2OS/ADM or MG63/ADM cells by JC-1 assay followed by flow cytometry analysis. Cells were transfected with siRNA targeting IncARSR. Twenty-four hours later, cells were exposed to $2 \mu \mathrm{g} / \mathrm{ml}$ ADM, and apoptosis was measured at different time-points. ${ }^{*} P<0.05$, vs. Si-NC; ${ }^{\#} P<0.05$, vs. si-NC plus ADM. e The indicated genes expression in U2OS/ADM and MG63/ADM cells in the presence or absence of ADM were detected by western blot, separately. $\mathbf{f}$ The indicated genes expression in MG63/ADM cells in the presence or absence of ADM were detected by western blot and real-time PCR, separately. si-NC, siRNA negative control. Data obtained from at least three independent experiments and presented as means plus standard deviation.

Furthermore, lncARSR-knockdown cells were exposed to increasing concentrations of ADM, and the Rh123 accumulation was accessed. The results in Fig. 4b indicated that si-lncARSR promoted Rh123 retention in an ADM dose-dependent manner. To observe the effects of lncARSR on cell motility, we conducted transwell assay without matrigel. Figure 4c proved that attenuation of lncARSR repressed cell migration significantly. In addition, lncARSR knockdown enhanced ADM-triggered apoptosis in a time-dependent manner (Fig. 4d). To examine the details of lncARSR-induced alterations, we measured the expression of MRP1, Survivin, and MMP2 was detected by immunoblots and qRT-PCR, respectively. As the results shown in Fig. 4e, the diminishment of IncARSR repressed the expression of MRP1, Survivin, and MMP2. In line with the changes of protein, the expression of lncARSR and the interesting mRNA declined significantly in cells expressing si-lncARSR (Fig. 4f).

Hindrance of IncARSR expression obstructs tumor growth and restores ADM-induced growth inhibition in U2OS/ ADM human osteosarcoma mouse models

To evaluate the potential effects of si-lncARSR plus ADM on tumor growth, we subcutaneously injected $\mathrm{U} 2 \mathrm{OS} / \mathrm{ADM}$ cells to $\mathrm{BALB} / \mathrm{c}$ nude mice to generate ADM-resistant osteosarcoma mouse models. Mice harbored approximately $100-\mathrm{mm}^{3}$ tumors received $6 \mathrm{mg} / \mathrm{kg}$ $\mathrm{ADM}$ or equal saline by intraperitoneal injection once a week according to the previous protocols ${ }^{19}$. The tumor volumes were measured every 4 days, as well as the body weights. Figure 5 a demonstrated that the deregulation of IncARSR suppressed tumor growth significantly compared to siRNA negative control. Importantly, si-lncARSR recovered the suppression of tumor growth by ADM. The alterations of tumor weights were consistent with those of tumor volumes (Fig. 5b). The increment of body weights among divergent groups showed no significant difference (Fig. 5c), suggested that the doses of the combination regimens were tolerant. The MRP1 expression in tumors was accessed by immunohistochemistry further. Figure $5 \mathrm{~d}$ illuminated the MRP1 expression reduced notably in tumors deregulated lncARSR compared to that in tumors with siRNA negative control. Consistent with the previous in vitro experiments, the expression of MRP1, Survivin, and MMP2 fell remarkably in tumors without lncARSR (Fig. 5e and f).

\section{Interference of IncARSR reverses resistance to ADM and represses tumor malignancy via hindering AKT activation}

We investigated the phosphorylation of the frequently hyperactivated kinases in osteosarcoma, including PI3K/ $\mathrm{AKT}^{20}, \mathrm{MAPK} / \mathrm{ERK}^{21}, \beta$-Catenin ${ }^{22}$, and $\mathrm{NFKB}^{23}$. The results in Fig. 6a displayed that the phosphorylation of AKT in U2OS/ADM xenograft models dropped, while the expression of total AKT changed little. Meanwhile, the activation of ERK, $\beta$-Catenin, and NFKB changed hardly. The pan-PI3K inhibitor BKM120 was subsequently introduced into the ADM-resistant sublines. Figure $6 \mathrm{~b}$ showed that the inhibition of AKT caused dephosphorylation of mTOR (Mammalian Target of Rapamycin), which was the direct target of AKT and played a central role in the regulation of cellular growth and survival. Moreover, the expression of MRP1, Survivin, and MMP2 reduced, accompanied by the deregulation of AKT. In line with expectations, the alterations of AKT and mTOR at mRNA level were consistent with that at protein level (Fig. 6c). The exposure of BKM120 suppressed cellular growth (Fig. 6d) and rhodamine 123 cellular retention (Fig. 6e), whereas promoting apoptosis (Fig. 6f). The migration of ADM-resistant sublines decreased post-treatment of BKM120 as well (Fig. 6g). The mechanisms that lncARSR conferred ADM resistance and promoted osteosarcoma progression via activating AKT were summarized in Fig. $6 \mathrm{~h}$.

\section{Discussion}

The most common cancer of bone in children and adults, osteosarcoma, is generally controlled successfully by surgery combined radiotherapy and adjuvant chemotherapy when it is localized. Adriamycin, together with methotrexate and cisplatin, form the backbone of chemotherapy, which commonly termed as "MAP." Although various active compounds have supplemented conventional chemotherapy, the outcomes for patients with 

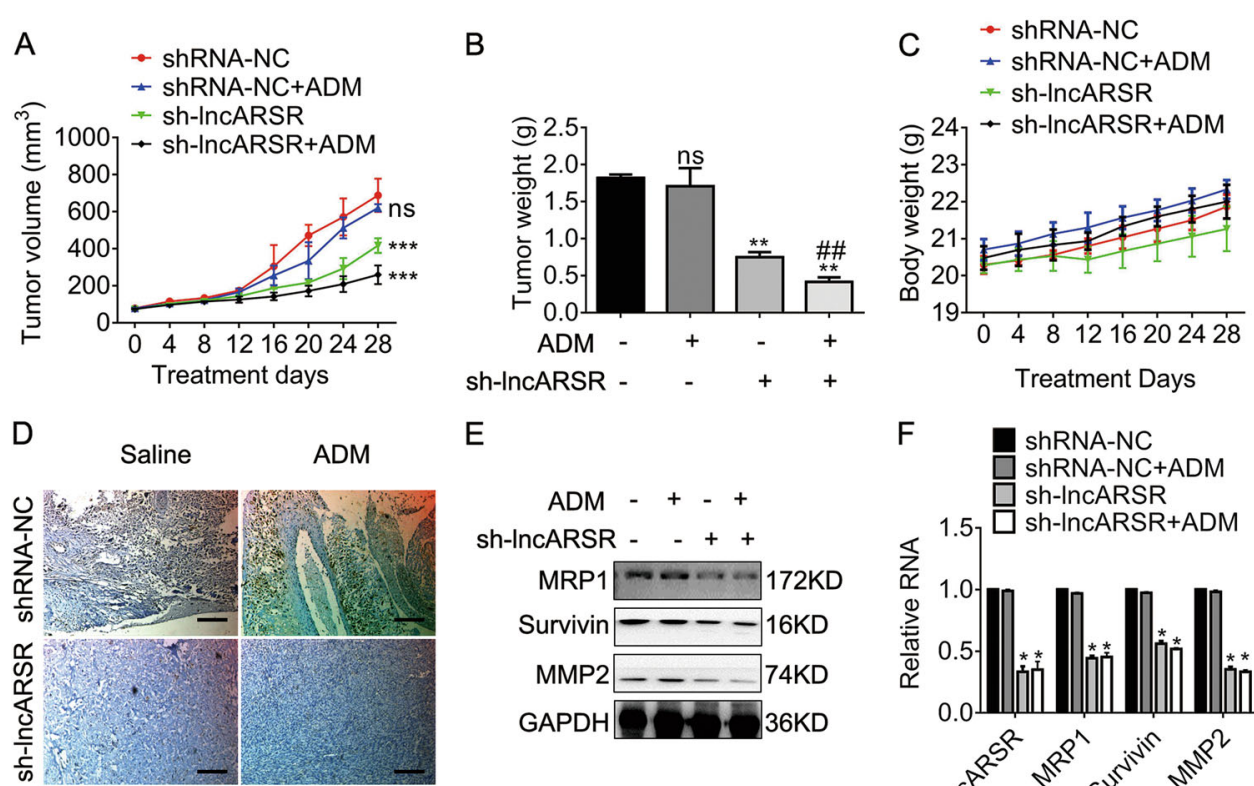

E

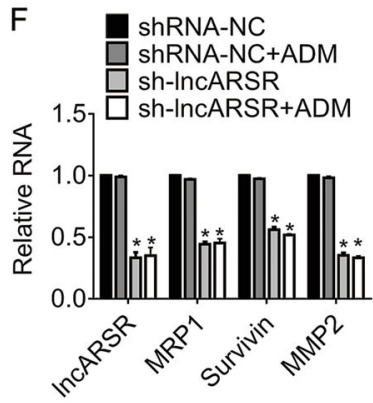

Fig. 5 The knockdown of IncARSR suppresses tumor growth via promoting sensitivity to ADM. a Tumor volumes of U2OS/ADM mouse models. Mouse in each group received $6 \mathrm{mg} / \mathrm{kg}$ ADM or equal volumes of saline by intraperitoneal injection once per week. *** $P<0.001$, vs. shRNANC. ns, no significance, vs. shRNA-NC. $\mathbf{b}$ Tumor weights of U2OS/ADM mouse models 28 days after treatment with ADM. ${ }^{*} P<0.01$, vs. shRNA-NC. ns, no significance, vs. shRNA-NC. ${ }^{\# \# P}<0.01$, vs. sh-IncARSR. c Body weights of U2OS/ADM mouse models. d Representative images for immunohistochemical staining of MRP1. Scale bar, $200 \mu \mathrm{m}$. Magnification, $\times 100$. e The indicated protein expression in tumors was accessed by immunoblot. $\mathbf{f}$ The expression of the indicated InCRNA and mRNA in tumors was measured by qRT-PCR. ${ }^{*} P<0.05$, vs. shRNA-NC. shRNA-NC, shRNA negative control. Data obtained from at least three independent experiments and presented as means plus standard deviation.

osteosarcoma have not increased for decades ${ }^{24,25}$. Apart from the limited response and adaptive resistance, the acquisition of cross-resistance to multiple active agents contributes to hinder the improvement of MAP-based chemotherapies. We observed that ADM-resistant osteosarcoma cells gained the resistance to paclitaxel and cisplatin, suggesting both U2OS/ADM and MG63/ ADM cells developed MDR phenotypes. Consistent with the results, we found that rhodamine 123 accumulation of ADM-resistant cells fell significantly in comparison with the corresponding parental cells. It is well known that $\mathrm{P}$ glycoprotein (ABCB1), MRP1 (ABCC1), MRP2 (ABCC2), and breast cancer resistance protein (ABCG2) played pivotal roles in the regulation of MDR. MRP1, belonging to the superfamily of ATP-binding cassette transporters and conferring resistance to anticancer compounds by exporting drugs in cells $^{26}$, was the most increased expression in these protein. Hence, we selected MRP1 to carried out the following exploration.

An increasing number of studies have illustrated that IncRNA was involved in the regulation of various pathogenesis process in cancer, including cell growth, proliferation, apoptosis, motility, and chemoresistance ${ }^{3,27}$. Notably, several studies have found lncRNA contributes to chemoresistance via multiple signaling cascades in osteosarcoma. Our study found the lncARSR upregulated in ADM-resistant cells and conferred MDR by promoting MRP1 and P-gp expression. A previous study supported our findings, which has shown that IncLUCAT1 promoted MDR related genes MDR1 and MRP5 by sponging miR-200c, conferring resistance to methotrexate ${ }^{28}$. Furthermore, Cheng et al. reported that lncROR triggered P-gp expression, lading to resistance to cisplatin ${ }^{29}$. Conversely, upregulated IncRNA FENDRR expression deprived resistance against $\mathrm{ADM}$ via suppressing the expression of P-gp and MRP1 ${ }^{4}$. The conflict issues urge more details to clarify the complex roles of lncRNA in the regulation of chemoresistance.

We observed the diminishment of lncARSR inhibited tumor growth and conquered ADM resistance in vivo. The evidence validated the findings in vitro. Prior studies have demonstrated that lncARSR activated AKT through diverse mechanisms. $\mathrm{Qu} \mathrm{L}$ et al. revealed that lncARSR promoted AKT activation indirectly and led to Sunitinib resistance of renal cancer ${ }^{10}$. Li and colleagues, as well as Ying $J$ et al., proved that lncARSR activated AKT via suppressing PTEN directly ${ }^{13,30}$ Furthermore, IncARSR enhanced AKT activation by promoting YAP1/IRS2/AKT signaling pathways ${ }^{31}$. It is well documented that AKT activation was involved in chemoresistance in various cancer, including ovarian and endometrial cancer, breast cancer, non-small cell lung cell, and osteosarcoma ${ }^{32}$. 


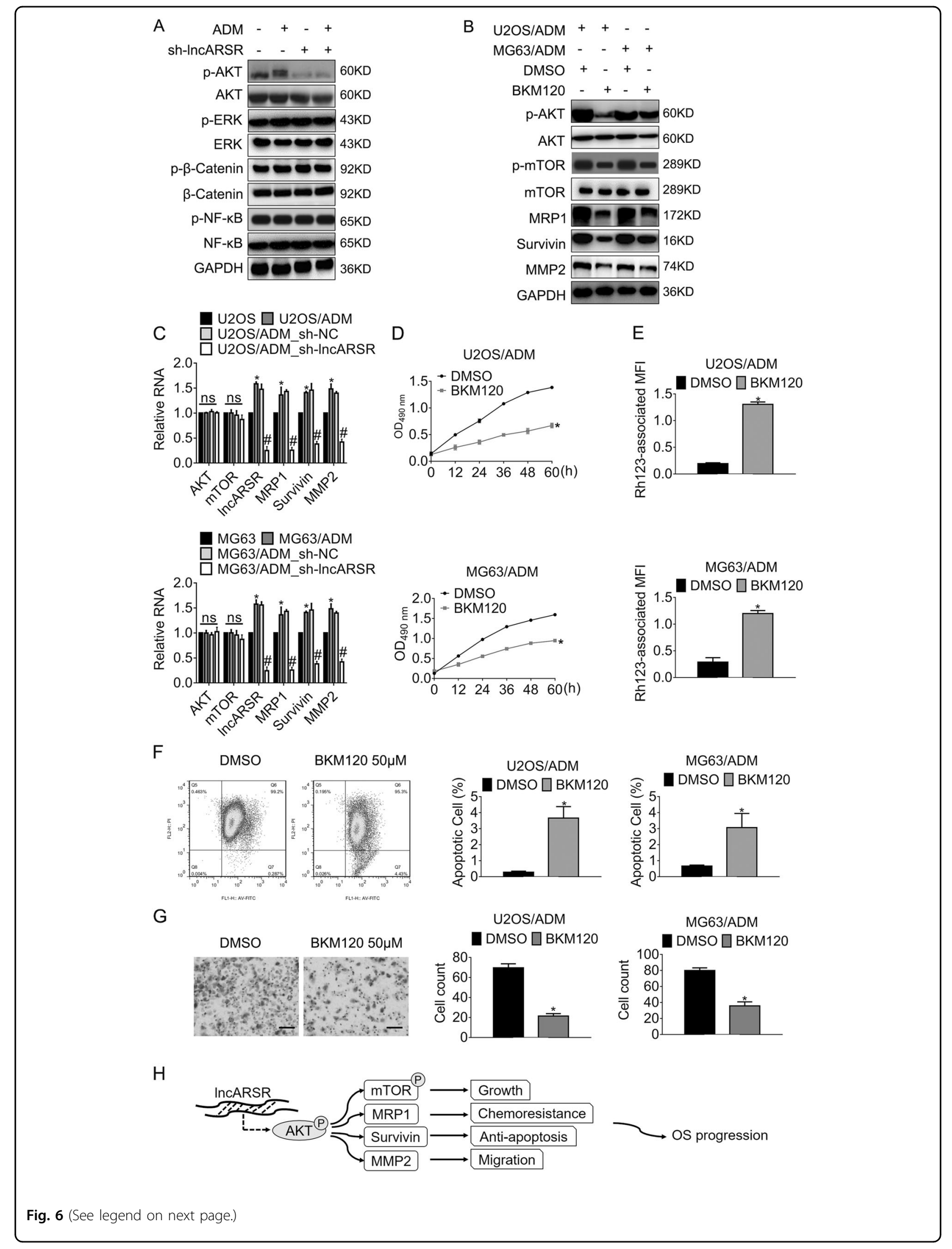


(see figure on previous page)

Fig. 6 Interference of IncARSR impedes resistance against ADM and retards tumor progression in an Akt-dependent manner. a The expression of the indicated protein in U2OS/ADM was detected by western blot. $\mathbf{b}$ The expression of the indicated protein in ADM-resistant cells was detected by western blot. c The expression of the indicated genes was examined by qRT-PCR. ${ }^{*} P<0.05$, vs. parental cells. ${ }^{*} P<0.05$, vs. ADM-resistant cells with sh-NC. $\mathbf{d}$ The growth of the indicated cells was accessed by MTT assay. ${ }^{*} P<0.05$, vs. DMSO. e Rh123 retention of the indicated cells was accessed by rhodamine 123 assay, followed by flow cytometry analysis. ${ }^{*} P<0.05$, vs. DMSO. $\mathbf{f}$ The apoptosis of the indicated cells was analyzed by JC1 assay and flow cytometry analysis. ${ }^{*} P<0.05$, vs. DMSO. $\mathbf{g}$ The migration of the indicated cells was accessed by transwell assay. ${ }^{*} P<0.05$, vs. DMSO. Scale bar, $200 \mu \mathrm{m}$. Magnification, $\times 100$. $\mathbf{h}$ The schematic pathways of IncARSR confers ADM resistance and promotes OS malignancy via activating AKT-mediated cascades. The aberrant expression of IncARSR activates AKT, subsequently enhances mTOR phosphorylation and the expression of MRP1, Survivin, and MMP2, leading to cell growth, acquisition of chemoresistance, survival, and migration. ADM, Adriamycin. OS, osteosarcoma. Cells were exposed to pan-PI3K inhibitor BKM120 $50 \mu \mathrm{M}$ for $24 \mathrm{~h}$ before analysis. Data obtained from at least three independent experiments and presented as means plus standard deviation.

For example, hypoxia-activated AKT led to overexpression of MRP1 in hepatocellular carcinoma $^{33}$. Transgelin2 motivated AKT and enhanced MRP1 via GSK-3 $\beta$-dependent pathways in breast cancer ${ }^{34}$. Aberrant expression of glucose transporter 1 enhanced phosphorylation of AKT and promoted Survivin expression, inducing chemoresistance of triplicate-negative breast cancer ${ }^{35}$. Smad4 diminished colorectal cancer cells resistance to 5'-fluorouracil by repressing AKT-Survivin cascades $^{36}$. Activation of AKT triggered MMP2 via various signaling pathways, such as EGFR-AKT-MMP2 $2^{37}$ and AKT-mTOR-MMP2 ${ }^{38}$. The evidence provided a rationale for investigating the effects of AKT-targeted therapy on overcoming the chemoresistance of osteosarcoma. Moreover, the selective PI3K inhibitor BKM120 conquered resistance to chemotherapy by suppression of AKT axis and MDR expression ${ }^{39}$. Many preclinical studies have confirmed the efficiency of PI3K/AKT/mTOR pathways-targeted chemicals on chemo-resistant osteosarcoma; however, only a few mTOR-targeted compounds succeeded in entering clinical trials currently. The conflict between the success of bench studies and the failure of clinical trials indicated the complex network of AKTmodulated chemoresistance remained largely unknown. Our findings that lncARSR conferred ADM resistance and boosted osteosarcoma malignancy through AKTdependent pathways, at least, fulfilled parts of the gaps between lncARSR and AKT-mediated chemoresistance. Exploration of lncARSR-targeted regimen and AKT inhibitors combination therapy may help improve the clinical outcomes of recurrent or refractory osteosarcoma.

More details are needed to clarify the connection between lncARSR and the clinical-pathological features. First, the distribution of lncARSR in stage-specific osteosarcoma or recurrent/metastatic osteosarcoma remains unknown. Second, the relationship between lncARSR and oncogenes in AKT-related cascades, such as $R A S, R A F$, or $S R C$, is a lack of investigation. Third, patient-derived xenograft models are necessary for further evaluating the potential of combination regimens.
Briefly, the current study demonstrated the lncARSR increased in ADM-resistant osteosarcoma cells, induced overexpression of MRP1, Survivin, and MMP2 via activating AKT. Deregulation of lncARSR recovered the sensitivity to ADM. Furthermore, combined sh-lncARSR and ADM magnified the inhibition of tumor growth. Our study extended the knowledge about the involvement of lncARSR in chemoresistance and provided a novel therapeutic target for treating refractory osteosarcoma.

Conflict of interest

The authors declare that they have no conflict of interest.

\section{Publisher's note}

Springer Nature remains neutral with regard to jurisdictional claims in published maps and institutional affiliations.

Received: 15 January 2020 Revised: 27 April 2020 Accepted: 28 April 2020 Published online: 13 May 2020

\section{References}

1. Rastogi, S., Aggarwal, A., Tiwari, A. \& Sharma, V. Chemotherapy in nonmetastatic osteosarcoma: recent advances and implications for developing countries. J. Glob. Oncol. 4, 1-5 (2018).

2. Isakoff, M. S., Bielack, S. S., Meltzer, P. \& Gorlick, R. Osteosarcoma: current treatment and a collaborative pathway to success. J. Clin. Oncol. 33, 3029-3035 (2015).

3. Li, Z., Dou, P., Liu, T. \& He, S. Application of long noncoding RNAs in osteosarcoma: biomarkers and therapeutic targets. Cell Physiol. Biochem. 42, 1407-1419 (2017).

4. Kun-Peng, Z., Xiao-Long, M. \& Chun-Lin, Z. LncRNA FENDRR sensitizes doxorubicin-resistance of osteosarcoma cells through down-regulating $A B C B 1$ and ABCC1. Oncotarget 8, 71881-71893 (2017).

5. Wang, Z., Liu, Z. \& Wu, S. Long non-coding RNA CTA sensitizes osteosarcoma cells to doxorubicin through inhibition of autophagy. Oncotarget $\mathbf{8}$, 31465-31477 (2017).

6. Wang, Y. et al. Long non-coding RNA LINC00161 sensitises osteosarcoma cells to cisplatin-induced apoptosis by regulating the miR-645-IFIT2 axis. Cancer Lett. 382, 137-146 (2016).

7. Xia, W. K. et al. Clinical implication of long noncoding RNA $91 \mathrm{H}$ expression profile in osteosarcoma patients. Onco Targets Ther. 9, 4645-4652 (2016).

8. Dong, Y. et al. MALAT1 promotes the proliferation and metastasis of osteosarcoma cells by activating the PI3KJAkt pathway. Tumour Biol. 36, 1477-1486 (2015).

9. Pasic, I. et al. Recurrent focal copy-number changes and loss of heterozygosity implicate two noncoding RNAs and one tumor suppressor 
gene at chromosome $3 q 13.31$ in osteosarcoma. Cancer Res. 70, 160-171 (2010).

10. Qu, L. et al. Exosome-transmitted IncARSR promotes sunitinib resistance in renal cancer by acting as a competing endogenous RNA. Cancer Cell $\mathbf{2 9}$ 653-668 (2016).

11. Qu, L. et al. A feed-forward loop between IncARSR and YAP activity promotes expansion of renal tumour-initiating cells. Nat. Commun. 7, 12692 (2016).

12. Zhang, M., Chi, X., Qu, N. \& Wang, C. Long noncoding RNA IncARSR promotes hepatic lipogenesis via Akt/SREBP-1c pathway and contributes to the pathogenesis of nonalcoholic steatohepatitis. Biochem. Biophys. Res. Commun. 499, 66-70 (2018).

13. Li, Y., Ye, Y., Feng, B. \& Qi, Y. Long noncoding RNA IncARSR PRomotes Doxorubicin Resistance in Hepatocellular Carcinoma Via Modulating PTEN-PI3K Akt pathway. J. Cell Biochem. 118, 4498-4507 (2017).

14. Yang, C. et al. IncARSR promotes liver cancer stem cells expansion via STAT3 pathway. Gene 687, 73-81 (2019).

15. Jouan, E. et al. Evaluation of P-glycoprotein inhibitory potential using a Rhodamine 123 accumulation assay. Pharmaceutics 8, https://doi.org/10.3390/ pharmaceutics8020012 (2016).

16. Riss, T. L. et al. in Assay Guidance Manual (eds Sittampalam, G. S. et al.) (2004).

17. Livak, K. J. \& Schmittgen, T. D. Analysis of relative gene expression data using real-time quantitative $P C R$ and the 2(-Delta Delta $C(T))$ method. Methods $\mathbf{2 5}$, 402-408 (2001).

18. Karkare, S. et al. Detection and targeting insulin growth factor receptor type 2 (IGF2R) in osteosarcoma PDX in mouse models and in canine osteosarcoma tumors. Sci. Rep. 9, 11476 (2019).

19. Liu, W. et al. Dioscin inhibits stem-cell-like properties and tumor growth of osteosarcoma through Akt/GSK3/beta-catenin signaling pathway. Cell Death Dis. 9, 343 (2018).

20. Dai, H. et al. RanBP9/TSSC3 complex cooperates to suppress anoikis resistance and metastasis via inhibiting Src-mediated Akt signaling in osteosarcoma. Cell Death Dis. 7, e2572 (2016).

21. He, C., Sun, J., Liu, C., Jiang, Y. \& Hao, Y. Elevated H3K27me3 levels sensitize osteosarcoma to cisplatin. Clin. Epigenetics 11, 8 (2019).

22. Nomura, M. et al. Tegavivint and the beta-catenin/ALDH AXis in Chemotherapy-resistant and Metastatic Osteosarcoma. J. Natl Cancer Inst. 111 1216-1227 (2019).

23. Kim, M. et al. GFRA1 promotes cisplatin-induced chemoresistance in osteosarcoma by inducing autophagy. Autophagy 13, 149-168 (2017).

24. Marina, N. M. et al. Comparison of MAPIE versus MAP in patients with a poor response to preoperative chemotherapy for newly diagnosed high-grade osteosarcoma (EURAMOS-1): an open-label, international, randomised controlled trial. Lancet Oncol. 17, 1396-1408 (2016).
25. Gronchi, A. et al. Histotype-tailored neoadjuvant chemotherapy versus standard chemotherapy in patients with high-risk soft-tissue sarcomas (ISG-STS 1001): an international, open-label, randomised, controlled, phase 3, multicentre trial. Lancet Oncol. 18, 812-822 (2017).

26. Stride, B. D. et al. Pharmacological characterization of the murine and human orthologs of multidrug-resistance protein in transfected human embryonic kidney cells. Mol. Pharm. 52, 344-353 (1997).

27. Yang, Z. et al. Long noncoding RNAs in the progression, metastasis, and prognosis of osteosarcoma. Cell Death Dis. 7, e2389 (2016).

28. Han, Z. \& Shi, L. Long non-coding RNA LUCAT1 modulates methotrexate resistance in osteosarcoma via miR-200c/ABCB1 axis. Biochem Biophys. Res. Commun. 495, 947-953 (2018).

29. Cheng, F. H., Zhao, Z. S. \& Liu, W. D. Long non-coding RNA ROR regulated $A B C B 1$ to induce cisplatin resistance in osteosarcoma by sponging miR-1533p. Eur. Rev. Med. Pharm. Sci. 23, 7256-7265 (2019).

30. Ying, J., Yang, J. \& Liu, Y. LncARSR promotes non-small-cell lung cancer progression via regulating PTEN/Akt. Am. J. Transl. Res. 12, 857-866 (2020).

31. Chi, Y., Gong, Z., Xin, H., Wang, Z. \& Liu, Z. Long noncoding RNA IncARSR promotes nonalcoholic fatty liver disease and hepatocellular carcinoma by promoting YAP1 and activating the IRS2/AKT pathway. J. Transl. Me.d 18, 126 (2020).

32. Zheng, $\mathrm{H}$. C. The molecular mechanisms of chemoresistance in cancers. Oncotarget 8, 59950-59964 (2017).

33. Xie, Y. \& Zhong, D. W. AEG-1 is associated with hypoxia-induced hepatocellular carcinoma chemoresistance via regulating PI3K/AKT/HIF-1alpha/MDR-1 pathway. EXCLI J. 15, 745-757 (2016).

34. Liu, L. et al. Transgelin 2 promotes paclitaxel resistance, migration, and invasion of breast cancer by directly interacting with PTEN and activating PI3K Akt/GSK-3beta pathway. Mol. Cancer Ther. 18, 2457-2468 (2019).

35. Oh, S., Kim, H., Nam, K. \& Shin, I. Silencing of Glut1 induces chemoresistance via modulation of Akt/GSK-3beta/beta-catenin/survivin signaling pathway in breast cancer cells. Arch. Biochem. Biophys. 636, 110-122 (2017).

36. Zhang, B. et al. Smad4 sensitizes colorectal cancer to 5-fluorouracil through cell cycle arrest by inhibiting the PI3K/Akt/CDC2/survivin cascade. Oncol. Rep. 35, 1807-1815 (2016)

37. $\mathrm{Xu}, \mathrm{H}$. et al. HCRP1 downregulation confers poor prognosis and induces chemoresistance through regulation of EGFR-AKT pathway in human gastric cancer. Virchows Arch. 471, 743-751 (2017).

38. He, K, Duan, G. \& Li, Y. Dehydroeffusol inhibits viability and epithelialmesenchymal transition through the Hedgehog and Akt/mTOR signaling pathways in neuroblastoma cells. Eur. J. Pharm. 829, 93-101 (2018).

39. Hu, Y. et al. Effects of PI3K inhibitor NVP-BKM120 on overcoming drug resistance and eliminating cancer stem cells in human breast cancer cells. Cell Death Dis. 6, e2020 (2015). 